\title{
Quality Characteristics and Anthocyanin Profiles of Different Vitis amurensis Grape Cultivars and Hybrids from Chinese Germplasm
}

\author{
Lei Zhu ${ }^{1,2,3,4}$, Xinyue $\mathrm{Li}^{1}{ }^{1}$ Xixi $\mathrm{Hu}^{5}{ }^{5}$, Xin $\mathrm{Wu}^{1}{ }^{1}$, Yunqing Liu ${ }^{1}$, Yiming Yang ${ }^{6}$, Yanqing Zang ${ }^{1,3} \mathbb{D}^{\mathbb{D}}$, \\ Huacheng Tang ${ }^{1}$, Changyuan Wang ${ }^{1,3, *}$ and Jingyu $X u^{3,7, * \mathbb{D}}$
}

1 College of Food Science and Technology, Heilongiiang Bayi Agricultural University, Daqing 163319, China; zhulei2580@126.com (L.Z.); lixinyue3676@163.com (X.L.); wuxin12021@163.com (X.W.); liuyunqing0515@163.com (Y.L.); byndzangyanqing@163.com (Y.Z.); byndthc@126.com (H.T.)

2 Quality Supervising and Testing Center of Ministry of Agriculture and Rural Affairs for Agricultural Products and Processed Goods, Daqing 163319, China

3 Department of National Coarse Cereals Engineering Research Center, Daqing 163319, China

4 Agri-Food Processing and Engineering Technology Research Center of Heilongjiang Province, Daqing 163319, China

5 Daqing Branch, Heilongjiang Academy of Agricultural Sciences, Daqing 163319, China; huxixi116@163.com

6 Institute of Special Animal and Plant Sciences of Chinese Academy of Agricultural Sciences,

Changchun 130112, China; yangyiming@caas.cn

7 College of Agriculture, Heilongjiang Bayi Agricultural University, Daqing 163319, China

* Correspondence: byndwcy@163.com (C.W.); xujingyu2003@hotmail.com (J.X.)

Citation: Zhu, L.; Li, X.; Hu, X.; Wu, X.; Liu, Y.; Yang, Y.; Zang, Y.; Tang, H.; Wang, C.; Xu, J. Quality

Characteristics and Anthocyanin Profiles of Different Vitis amurensis Grape Cultivars and Hybrids from Chinese Germplasm. Molecules 2021, 26, 6696. https://doi.org/10.3390/ molecules 26216696

Academic Editor:

Domenico Trombetta

Received: 8 September 2021

Accepted: 27 October 2021

Published: 5 November 2021

Publisher's Note: MDPI stays neutral with regard to jurisdictional claims in published maps and institutional affiliations.

Copyright: (c) 2021 by the authors. Licensee MDPI, Basel, Switzerland. This article is an open access article distributed under the terms and conditions of the Creative Commons Attribution (CC BY) license (https:// creativecommons.org/licenses/by/ $4.0 /)$
Abstract: To evaluate the important Vitis amurensis germplasm, the quality characteristics and anthocyanin profiles of the ripe berries of $20 \mathrm{~V}$. amurensis grapes and 11 interspecific hybrids in two consecutive years were analysed. Compared with the $V$. vinifera grapes, $V$. amurensis grapes had small berries with low total soluble solids and high titratable acids, and were richer in phenolic compounds except for flanan-3-ols in their skins but had lower phenolic contents in their seeds and showed lower antioxidant activities. An outstanding feature of the V. amurensis grapes was their abundant anthocyanin contents, which was 8.18-fold higher than the three wine grapes of $V$. vinifera. The anthocyanin composition of $V$. amurensis was characterized by an extremely high proportion of diglucoside anthocyanins (91.71\%) and low acylated anthocyanins (0.04\%). Interestingly, a new type of speculated 3,5,7-O-triglucoside anthocyanins was first identified and only detected in $V$. amurensis grapes and hybrids. Based on the total phenolic and anthocyanin characteristics, $V$. amurensis grapes were set apart from $V$. vinifera cultivars and the interspecific hybrids, for the same qualities, fell between them, as assessed by principal component analysis.

Keywords: Vitis amurensis; anthocyanin composition and content; total phenolic content; general characteristic; antioxidant activity

\section{Introduction}

Vitis amurensis Rupr., an important species belonging to the East-Asian population of Vitaceae, is mainly distributed in northeast China, Far East Russia and the Korean Peninsula [1]. The cold resistance of $V$. amurensis is the strongest among all the grape species. Its roots and branches can survive at extremely low temperatures of $-14--16{ }^{\circ} \mathrm{C}$ and $-45^{\circ} \mathrm{C}$, respectively [2]. And V. amurensis is highly resistant to white rot (Coniothyrium diplodiella), grape anthracnose (Glomerella cingulata) and grape bitter rot (Greeneria uvicola) [3]. These diseases have very adverse effects on Vitis vinifera grapes, which are widely cultivated and commercialized throughout the world. Thus, $V$. amurensis is one of the precious rootstock and breeding resources with resistance to cold and diseases in Vitis.

Since the late 1950s, the survey, collection and evaluation of $V$. amurensis germplasm resources have been systematically carried out in China [2]. Through decades of efforts, 
more than 400 resources are preserved in the V. amurensis germplasm repository (VAGR) of Chinese Academy of Agricultural Sciences (CAAS). Moreover, a technical cultivation system of $V$. amurensis cultivars and hybrids has been constructed and promoted in China [4]. In winter, vines do not need burying in most of northern China, which can greatly reduce the cost of cultivation, while the $V$. vinifera grapes can not be cultivated at all in the open fields of Heilongjiang, Jilin and northern Liaoning. Today, China has the largest area in the world cultivated by grapes with the $V$. amurensis pedigree [2]. Most $V$. amurensis resources yield small and red berries [5]. As a result, $V$. amurensis grapes and hybrids are mainly used to make red wines, including dry wines, sweet wines, ice wines and low-alcohol and non-alcoholic wines [5]. The wine pomace can also be used to produce health care products and additives. And a few interspecific hybrids are used as table grapes or juice grapes, in China. However, there are few reports of the quality characteristics of the V. amurensis germplasm in international academic journals.

There are rich sources of phenolic compounds in the grapes of $V$. amurensis, including phenolic acids, stibenes, anthocynins, flavonols and flavan-3-ols [6-9]. In a recent study, a total of 118 phenolic metabolites were detected in wild and cultivated V. amurensis grapes collected from Far East Russia [10]. The phenolic extracts from $V$. amurensis have been proven to possess antioxidant [11], anti-inflammatory [12] and antiproliferative [13,14] activities, which could attenuate degenerative processes such as aging [15], cancer [16] and cardiovascular disease [17]. More importantly, phenolic content and composition have significant influences on the quality of grape berries and wines. Among these phenolic compounds, the abundant content of anthocyanins is the most notable characteristic of $V$. amurensis [18] The literature contains several reports concerning $V$. amurensis anthocyanins from studies using high-performance liquid chromatography coupled with mass detection (HPLCMS) $[8,10,18-21]$;however, in these, the selected grape cultivars/accessions were neither comprehensive nor representative.

In this study, the important germplasm resources in VAGR of CAAS, including $20 \mathrm{~V}$. amurensis cultivars/accessions and 11 interspecific hybrids crossed with $\mathrm{V}$. vinifera, were collected to systematically and comprehensively investigate their quality characteristics and, especially, their anthocyanin profiles, and comparedwith 3 red wine grapes of $V$. vinifera. Analysis by ultra-performance liquid chromatography coupled with Qexactive orbitrap mass detection (UPLC/Q-Exactive orbitrap MS) was conductedon their anthocyanin profiles, which are more efficient and sensitive techniques in comparison with HPLC-ion trap MS. Rich anthocyanin compounds were found and a new type of anthocyanin was preliminarily identified in grapes of the $V$. amurensis pedigree.

\section{Material and Methods}

\subsection{Plant Material}

All the materials of $31 \mathrm{~V}$. amurensis cultivars/accessions and hybrids in this study were collected from the VAGR of CAAS. The materials of the three $V$. vinifera wine grapes were collected from the experimental vineyards of China Agricultural University (Table 1). All red berry samples were harvested upon ripening in two consecutive years, 2017 and 2018, determined based on their content of total soluble solids and as judged from seeds' color-change to dark brown without the senescence of berry tissue. At least 30 clusters were collected at 6 grapevines for each cultivar. The fresh clusters were surrounded by ice packs and immediately taken to the laboratory. Then, three 100-berry batches, with berries randomly selected from the top, middle and bottom portions of the clusters, were collected. Each batch was considered to be one sample, too allow for three experimental replications for each grape cultivar/accession. 
Table 1. Grape cultivars/accessions collected in this study.

\begin{tabular}{ccc}
\hline Pedigree & Number & Cultivars/Accessions \\
\hline Vitis amurensis & 20 & $\begin{array}{c}\text { Tonghua-1; Tonghua-3; Tonghua-7; Changbai-5; Changbai-8; Changbai-9; } \\
\text { 92; 14; 75081; 73102; 84001; 84002; 85010; 086919; Zuoshan-1; Zuoshan-2; } \\
\text { Shuangqing (Changbai-11); Shuangyou *; Shuanghong *; Shuangfeng * }\end{array}$ \\
$\begin{array}{ccc}\text { Hybrids of } & \text { Zuohong-1; Zuoyouhong; Beichun; Beihong; Beimei; Xuelanhong; } \\
\text { G. amurensis and V. vinifera } & 11 & \text { Cabernet Sauvignon; CabernetGernischt; Gem Cabernet }\end{array}$ \\
\hline V. vinifera & 3 & *: Intraspecific hybrids of V. amurensis.
\end{tabular}

\subsection{Chemicals and Standards}

Folin and Ciocalteu's phenol reagent ( $2 N)$, 2,2-diphenyl-1-picrylhy-drazyl (DPPH) ( $\geq 97 \%$ ), 2,2'-azino-bis-(3-ethylben zothiazoline-6-sulfonic acid) diammonium salt (ABTS) ( $\geq 98 \%$ ), 2,4,6-tripyridyl-s-triazine (TPTZ) ( $\geq 99 \%$ ) and 6-Hydroxy-2,5,7,8-tetramethylchroman-2-carboxylic acid (Trolox) ( $\geq 98 \%$ ) were obtained from Aladdin (Shanghai, China). The standards, gallic acid $(99 \%)$, catechin $(\geq 95 \%)$ and rutin $(98 \%)$, used in the colorimetric determination were purchased from Macklin (Shanghai, China), and the anthocyanin standards for UPLC-MS, malvidin-3-O-glucoside ( $\geq 90 \%)$ and malvidin-3,5-O-diglucosides $(\geq 90 \%)$ were obtained from Sigma-Aldrich (St. Louis, MO, USA). HPLC grade reagents, formic acid and acetonitrile were purchased from Fisher Scientific Co. (Fairlawn, NJ, USA). All other analytical-grade chemicals were purchased from Chinese Reagent Network (http:/ / www.labgogo.com/, accessed on: 1 November 2018).

\subsection{Determination of General Berry Characteristics}

Fifty fresh berries from each replicate sample were randomly selected to determine their general characteristics. The weights, transverse diameters and longitudinal diameters of the selected berries were recorded $(n=150)$. Approximately $20 \mathrm{~g}$ berries of eachbatch's 50 remaining berries were then weighed and crushed to assay their titratable acids $(n=3)$. The crushed volumes were diluted to $200 \mathrm{~mL}$ with distilled water. After centrifugation, $20 \mathrm{~mL}$ of supernatant was titrated with $0.1 \mathrm{~mol} / \mathrm{L} \mathrm{NaOH}$ to a $\mathrm{pH}$ endpoint of 8.1 [22], and the residue berries were crushed to directly determine the total soluble solid $(n=3)$ with a hand-held refractometer (Wancheng Co., Beijing, China) and the $\mathrm{pH}(n=3)$ with a $\mathrm{pH}$ meter (S210-B, METTLER TOLEDO, Zurich, Switzerland).

\subsection{Pretreatment and Extraction of Phenolic Compounds}

The skins and seeds were manually separated, freeze-dried (Alpha 1-4LSCbasic, Martin Christ Co., Osterode, Germany) and ground (BLF-YB2000, Bailifu Co., Shengzhen, China) into a fine powder. The ground seeds were defatted, twice, with petroleum ether for $3 \mathrm{~h}$. The final skin and seed samples were stored in vacuum-packaged polyethylene pouches at $-80^{\circ} \mathrm{C}$ for subsequent analysis.

A protocol developed in the previous work [23] was used for the extraction of phenolic compounds. Briefly, the solvents of methanol/water/acetic acid (70:29:1, v/v/v) was used for the skins, and methanol with $0.1 \%$ acetic acid for the seeds. The pulverized powder $(0.5 \mathrm{~g})$, mixed with $20 \mathrm{~mL}$ of extraction solvent, was shaking-extracted in a shaker (SHZ-88A, Taicang Experiment Equipment Factory, Taicang City, China) at room temperature for $2 \mathrm{~h}$. After centrifugation (GL-21M, Xiangyi Co., Hunan, China), the residues were re-extracted twice and thee extracts from each sample were collected together. The skin extracts for each replicate sample, after removing a volume of $2 \mathrm{~mL}$ for use in the colorimetric determination, was rotatory evaporated (RE-52A, Yarong Biochemistry Instrument Factory, Shanghai, China) at $30^{\circ} \mathrm{C}$ and redissolved in the extraction solvent to a unified volume of $5 \mathrm{~mL}$ for the anthocyanin analysis. 


\subsection{Determination of Total Contents of Phenolic Compounds}

The total phenol content (TP) was assayed with the Folin-Ciocalteu colorimetric method [24] with a UNICO UV-2100 spectrometer (UNICO, New York, NY, USA). The absorbances were converted to TP expressed as $\mathrm{mg}$ gallic acid equivalents (GAE)/g of dry weight (DM). The total flavonoid content (TFO) was measured with the $\mathrm{NaNO}_{2}-\mathrm{AlCl}_{3}$ method [25]. The TFO was expressed as mg rutin equivalents (RAE)/g DM, and total flavan-3-ols content (TFA) was determined by the Vanillin method [26] and expressed as mg catechin equivalents (CAE)/g DM.

\subsection{Analysis of Anthocyanin Compounds}

The anthocyanins in grape skins were analyzed using an Ultimate 3000 UPLC/QExactive orbitrap MS (Thermo Fisher Scientific, Waltham, MA, USA). The samples were diluted 10 times with an aqueous solution containing $10 \%$ acetonitrile and $2 \%$ formic acid and injected $(5 \mu \mathrm{L})$, directly after filtration and through a $0.45-\mu \mathrm{m}$ inorganic membrane, onto a Thermo GOLD HYPERSIL column $(\mathrm{C} 18,50 \mathrm{~mm} \times 2.1 \mathrm{~mm}, 1.9 \mu \mathrm{m})$ at $22{ }^{\circ} \mathrm{C}$. The solvent system consisted of an aqueous solution containing $2 \%$ formic acid (phase $\mathrm{A}$ ) and an acetonitrile solution containing $2 \%$ formic acid (phase B), and the flow rate was $0.3 \mathrm{~mL} / \mathrm{min}$. The gradient profile was from $2 \%$ to $10 \%$ B for $3 \mathrm{~min}$, from $10 \%$ to $20 \%$ B for $3 \mathrm{~min}$, isocratic $20 \%$ B for $1 \mathrm{~min}$, from $20 \%$ to $100 \%$ for $5 \mathrm{~min}$ and isocratic $100 \%$ for $2 \mathrm{~min}$. MS analysis was conducted using heating electrospray ionisation (HESI), positive ion model, a 13-psi nebulizer pressure, $35-\mathrm{mL} / \mathrm{min}$ dry gas flow rate, $300^{\circ} \mathrm{C}$ dry gas temperature, and $160-1000 \mathrm{~m} / z$ scan range. Monoglucoside and diglucoside anthocynins were quantified using malvidin-3-O-glucoside and malvidin-3,5-O-diglucoside as standards, respectively, and expressed as $\mu \mathrm{g}$ MGE or mg MGE per $g$ DM of grape skins.

\subsection{Determination of Antioxidant Activities}

The antioxidant activities of the phenolic extracts from grapes' skins and seeds were based on DPPH free-radical-scavenging ability [27], ABTS free-radical-scavenging ability [28] and ferric reducing/antioxidant power (FRAP) [29]. The results were expressed as $\mu \mathrm{mol}$ Trolox equivalents [30].

\subsection{Statistical Analysis}

All results were expressed as means \pm standard deviations (SD), as all the data of the variances were compatible with the normal distribution, but most were not homogeneous. The data from the same indicator between grape groups were subjected to Welch's analysis of variance (ANOVA) with the Games-Howell test at a 95\% confidence level. The correlations of different indicators were analyzed with Pearson correlation coefficient at a $95 \%$ confidence level. The principal component analysis (PCA) was performed to investigate the phenolic-based relationships of different grape cultivars/accessions. All the statistical analyses were conducted with SPSS 19.0 (SPSS Inc., Chicago, IL, USA).

\section{Results and Discussion}

\subsection{General Characteristics}

In general, the $V$. amurensis resources assayed in this study, including wild accessions/cultivars and intraspecific hybrids, had lighter and smaller berries with low total soluble solids and higher titratable acids. The three $V$. vinifera grapes with heavier and bigger berries produced higher total soluble solids and lower titratable acids. As expected, the interspecific hybrids were intermediate between V. amurensis and V. vinifera (Figure 1, Table S1); but there were also several exceptions. Among all the assayed grapes, the interspecific hybrid 'Huapu-1' had the weightiest and biggest berries. The intraspecific hybrids of V. amurensis, 'Shuanghong' and 'Shuangfeng', had higher total soluble solids and lower titratable acids than some interspecific hybrids. 

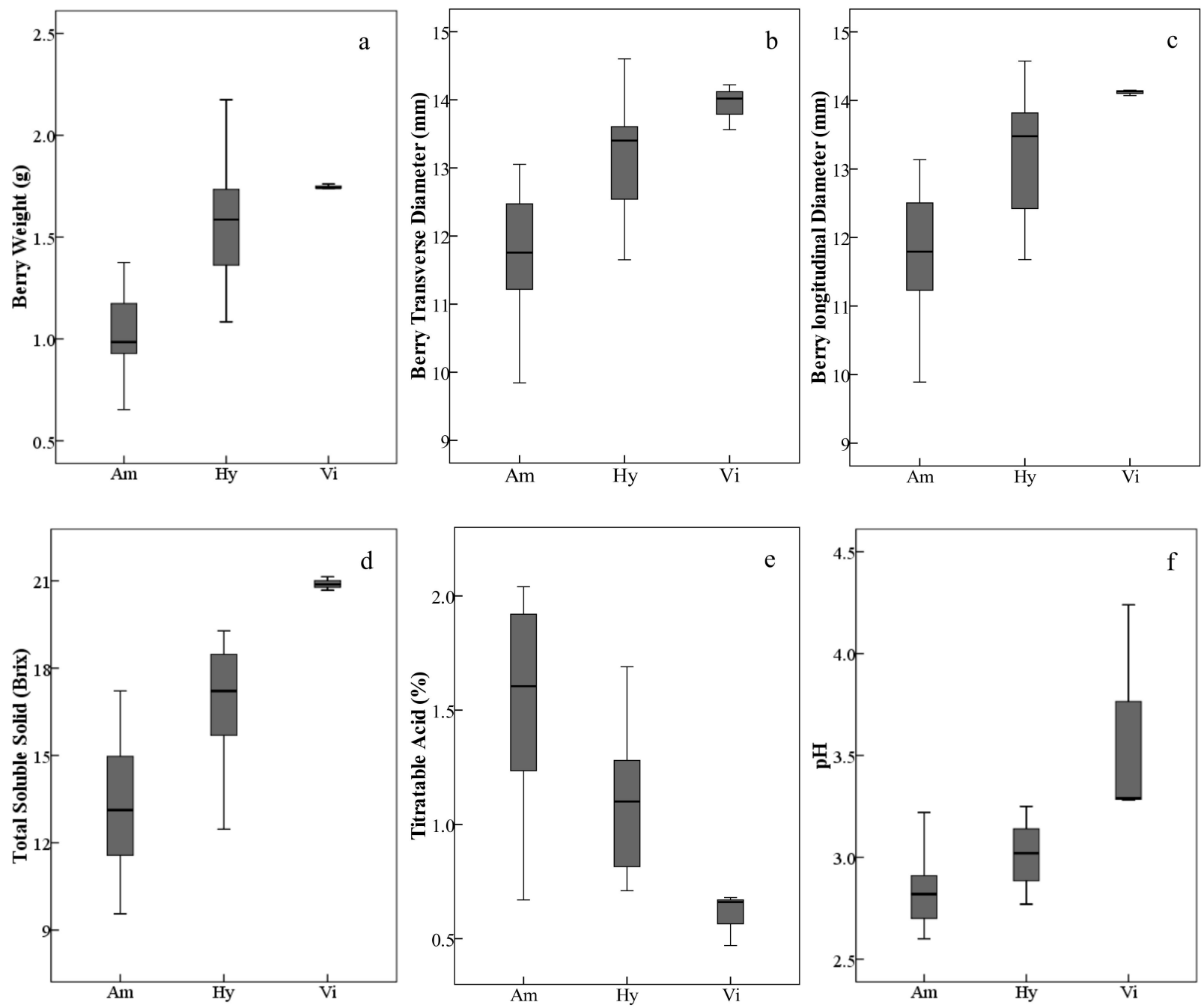

Figure 1. Ranges and distributions of weight (a), transverse diameter (b), longitudinal diameter (c), total soluble solid (d), titratable acid (e) and pH (f) of ripe berries belonging to different grape species/groups harvested in 2017 and 2018. The height of each box is equal to the interquartile range. The horizontal line in the interior of each box is the median. The whiskers extended from the top and bottom of each box with horizontal lines are the maximums and minimums, respectively. Abbreviations: Am, V. amurensis; Hy, hybrids of V. amurensis and V. vinifera; Vi, V. vinifera.

\subsection{Total Contents of Phenolic Compounds in Grape Skins and Seeds}

The average TP in grape skins of the three grape groups varied, and were, in decreasing order, $\mathrm{Am}>\mathrm{Vi}>\mathrm{Hy}$ (Figure 2a, Table S1). The TP in the skins of the grape cultivars/accessions of $V$. amurensis were generally higher. The accession ' 85010 ' had the highest TP (60.24 mg GAE/g DM) among all the grapes collected, followed by ' 14 ', ' 92 ', 'Tonghua- 1 ' and ' 086919 ', while the lowest TP was found in the interspecific hybrid 'Huapu-1' (16.45 mg GAE/g DM). Also, many interspecific hybrids possessed lower TP, namely 'Beihong', 'Xuelanhong', 'Gongniang-1' and 'Zuohong-1'. The TP in skins also varied significantly within the same group. For example, the V. amurensis accession '75081' had lower TP, which was similar with the interspecific hybrid 'Zuohong-1'. Similar distributions were found in the skin TFOs among all the grapes studied (Figure 2b, Table S1). However, the TFA in the skins of $V$. amurensis grapes and hybrids were significantly lower than in the three $V$. vinifera grape skins (Figure 2c, Table S1). 

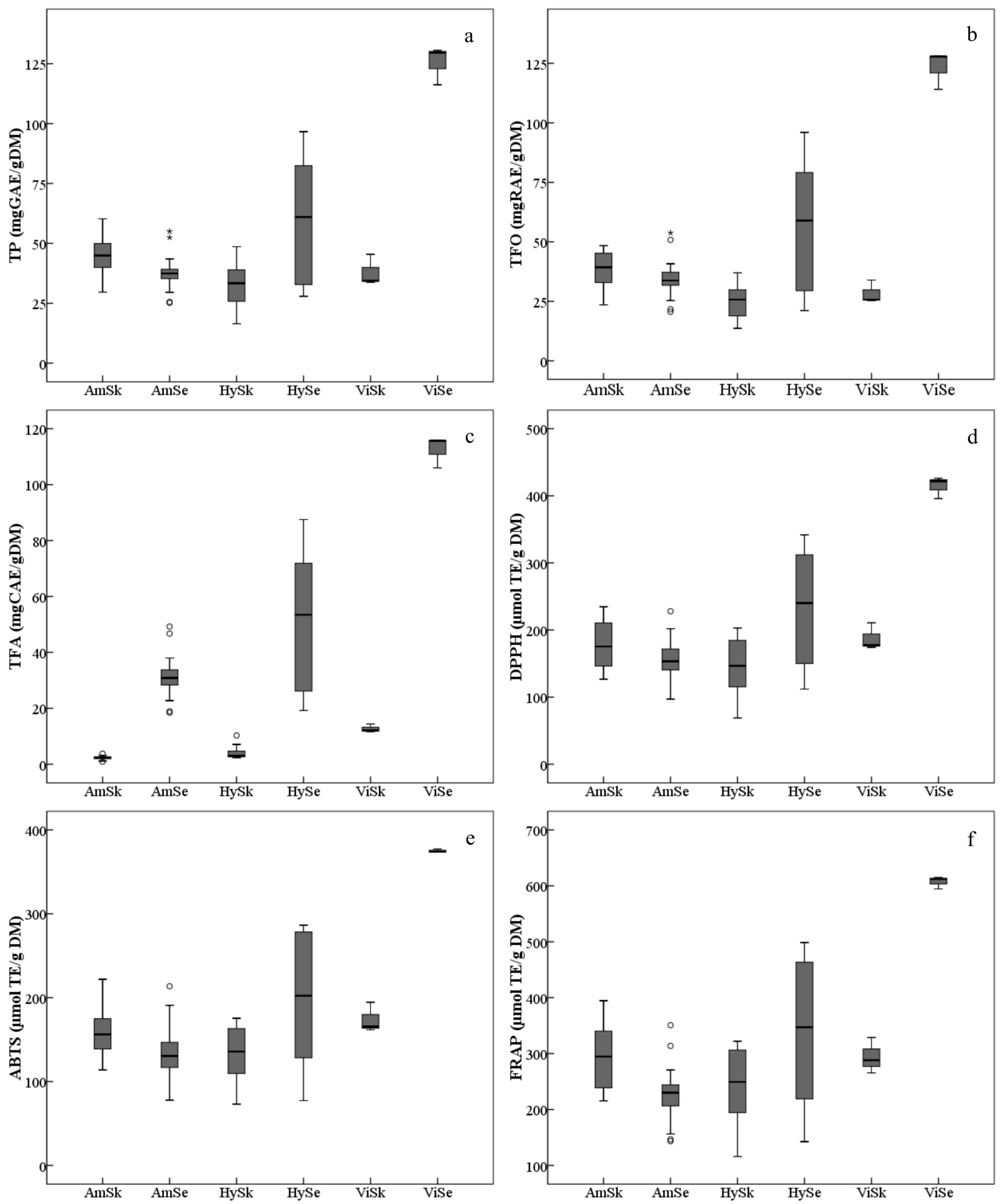

Figure 2. Ranges and distributions of total phenolics (TP, (a)), total flavonoids (TFO, (b)), total flavan-3-ols (TFA, (c)), DPPH free radical scavenging activities (DPPH, (d)), ABTS free radical scavenging activities (ABTS, (e)) and ferric ion/reducing antioxidant powers (FRAP, (f)) of ripe berry skins (Sk) and seeds (Se) belonging to the three grape groups from 2017 and 2018. The hollow dots and asterisks outside the whiskers indicate the mild and extreme outliers, respectively. The abbreviations of grape groups follow Figure 1.

The total content of phenolic compounds, including TP, TFO and TFA, in the seeds presented with consistent variation, by decreasing order of Vi $>\mathrm{Hy}>\mathrm{Am}$ (Figure $2 \mathrm{a}-\mathrm{C}$, Table S1). However, there were also significant differences among grape seeds within the 
same group. Taking the $V$. amurensis for example, the TP, TFO and TFA in the accessions ' 84,001 ' and '84,002' were significantly higher than those in other grapes, and were two-fold more than accessions ' 86,919 ' and ' 92 ', which had the least total phenolic contents.

\subsection{Anthocyanin Profiles}

\subsubsection{Identification of Anthocyanin Compounds}

A UPLC/Q-Exactive orbitrap MS was applied to the qualitative and quantitative analysis of anthocyanin compounds in this study. Compared with HPLC, UPLC can separate mixtures more efficiently so as to dramatically shorten the elution time in the chromatographic column, and the Q-Exactive orbitrap MS can detect more micro-components in samples. Combined with literature data [18,31,32], the compounds were identified by means of the extracted ion chromatograms obtained in $\mathrm{MS}^{2}$ mode.

A total of 48 anthocyanins were detected across all studied samples (Table 2). All the anthocyanins were glucoside derivatives of six anthocyanidins: delphinidin (Dp), cyanidin $(\mathrm{Cy})$, petunidin $(\mathrm{Pt})$, pelargonidin $(\mathrm{Pg})$, peonidin $(\mathrm{Pn})$ and malvidin $(\mathrm{Mv})$, which were dependent on the observed $m / z$ characteristic fragmentation values of 303, 287, 317, 271, 301 and 331, respectively. The simple glucoside derivatives, monoglucoside and diglucoside anthocyanins, were identified on the basis of their molecular ions $[\mathrm{M}]^{+}$as well as the fragment ions corresponding to the anthocyanidin after cleavage of one glucose unit $[\mathrm{M}-162]^{+}$and two glucose units $[\mathrm{M}-162]^{+}$and $[\mathrm{M}-324]^{+}$in $\mathrm{MS}^{2}$ mode, respectively. While the acylated derivatives, acetyl, $p$-coumaroyl and caffeoyl anthocyanins were confirmed by their molecular ions $[\mathrm{M}]^{+}$as well as the fragment ions corresponding to the anthocyanidin after cleavage of one esterified glucose moiety of $[\mathrm{M}-204]^{+},[\mathrm{M}-308]^{+}$and $[\mathrm{M}-324]^{+}$, in $\mathrm{MS}^{2}$ mode and respectively.

Table 2. Retention times, MS/MS $2 \mathrm{~m} / z$ values and mean contents ( $\mu \mathrm{g}$ MGE/g DM) of anthocyanin compounds detected in the skins of grape cultivars/accessions belonging to the three study groups by Q-Exactive orbitrap MS.

\begin{tabular}{|c|c|c|c|c|c|c|c|c|}
\hline \multirow{2}{*}{ Rt (min). } & \multirow{2}{*}{ MS; MS2 (m/z) } & \multirow{2}{*}{ Anthocyanin } & \multicolumn{2}{|c|}{ V. amurensis } & \multicolumn{2}{|c|}{ Interspecific Hybrids } & \multicolumn{2}{|c|}{ V. vinifera } \\
\hline & & & Mean & SE & Mean & SE & Mean & SE \\
\hline 2.41 & $789 ; 627,465,303$ & Dp-3,5,7-triglc * & 35.96 & 21.63 & 6.37 & 5.53 & - & - \\
\hline 2.97 & $773 ; 611,449,287$ & Cy-3,5,7-triglc * & 26.88 & 15.48 & 4.19 & 3.31 & - & - \\
\hline 3.01 & $627 ; 465,303$ & Dp-3,5-diglc & 4608.23 & 3395.62 & 321.30 & 352.87 & 15.79 & 19.66 \\
\hline 3.11 & $803 ; 641 ; 479,317$ & Pt-3,5,7-triglc * & 26.15 & 18.45 & 15.14 & 32.68 & - & - \\
\hline 3.48 & $611 ; 449,287$ & Cy-3,5-diglc & 3229.93 & 2843.26 & 311.09 & 406.38 & - & - \\
\hline 3.57 & $787 ; 625,463,301$ & Pn-3,5,7-triglc * & 7.34 & 6.81 & 8.69 & 7.60 & - & - \\
\hline 3.73 & $817 ; 655,493,331$ & Mv-3,5,7-triglc * & 681.46 & 1404.39 & 33.16 & 20.69 & - & - \\
\hline 3.72 & $641 ; 479,317$ & Pt-3,5-diglc & 3157.22 & 2087.73 & 388.53 & 321.75 & - & - \\
\hline 3.91 & $465 ; 303$ & Dp-3-glc & 861.17 & 824.13 & 1261.65 & 1607.40 & 148.58 & 194.19 \\
\hline 3.99 & $595 ; 433,271$ & Pg-3,5-diglc & 16.01 & 10.77 & 3.38 & 2.32 & - & - \\
\hline 4.18 & $625 ; 463,301$ & Pn-3,5-diglc & 3027.85 & 2968.72 & 802.29 & 562.95 & - & - \\
\hline 4.26 & $655 ; 493,331$ & Mv-3,5-diglc & $17,344.52$ & 6146.84 & 4273.00 & 2643.70 & 1.75 & 0.49 \\
\hline 4.4 & $669 ; 507,465,303$ & Dp-3-acglc-5-glc & 4.91 & 5.00 & 6.99 & 8.67 & - & - \\
\hline 4.42 & $449 ; 287$ & Cy-3-glc & 235.81 & 179.96 & 321.29 & 306.54 & 48.17 & 59.19 \\
\hline 4.7 & $479 ; 317$ & Pt-3-glc & 302.33 & 204.74 & 477.40 & 351.41 & 168.02 & 181.37 \\
\hline 4.96 & $433 ; 271$ & Pg-3-glc & 1.02 & 0.96 & 1.27 & 1.01 & 0.78 & 0.79 \\
\hline 5.09 & $653 ; 491,449,287$ & Cy-3-acglc-5-glc & 14.65 & 14.83 & 28.60 & 81.11 & - & - \\
\hline 5.09 & $683 ; 521,479,317$ & Pt-3-acglc-5-glc & 16.37 & 7.44 & 67.33 & 201.79 & - & - \\
\hline 5.17 & $463 ; 301$ & Pn-3-glc & 67.85 & 52.91 & 233.71 & 214.31 & 261.38 & 163.51 \\
\hline 5.34 & $493 ; 331$ & Mv-3-glc & 434.03 & 287.52 & 1350.04 & 867.34 & 2502.94 & 2426.91 \\
\hline 5.6 & $667 ; 505,463,301$ & Pn-3-acglc-5-glc & 15.93 & 17.60 & 6.84 & 3.98 & - & - \\
\hline 5.6 & $697 ; 535,493,331$ & Mv-3-acglc-5-glc & 71.59 & 38.15 & 32.77 & 27.89 & - & - \\
\hline 5.61 & $963 ; 801,639,493,331$ & Mv-3-cmglc-5-glc-7-glc * & - & - & 3.84 & 2.48 & - & - \\
\hline 5.76 & $789 ; 627,465,303$ & Dp-3-cfglc-5-glc & - & - & 17.65 & 39.97 & - & - \\
\hline 5.73 & $507 ; 303$ & Dp-3-acglc & 2.73 & 4.62 & 23.10 & 58.79 & 9.96 & 13.93 \\
\hline 6.17 & $803 ; 641,479,317$ & Pt-3-cfglc-5-glc & - & - & 9.02 & 15.20 & - & - \\
\hline 6.18 & $\begin{array}{l}773 ; 611,465,303 \\
/ 773 ; 611,449,287\end{array}$ & $\begin{array}{l}\text { Dp-3-cmglc-5-glc } \\
\text { /Cy-3-cfglc-5-glc }\end{array}$ & 10.19 & 25.14 & 213.59 & 636.50 & - & - \\
\hline 6.2 & $491 ; 287$ & Cy-3-acglc & 1.01 & 0.94 & 3.72 & 3.18 & 2.96 & 4.21 \\
\hline 6.33 & $521 ; 317$ & Pt-3-acglc & 0.88 & 0.64 & 10.93 & 13.32 & 21.02 & 25.23 \\
\hline 6.16 & $627 ; 303$ & Dp-3-cfglc & - & - & 0.67 & 1.14 & 0.18 & 0.23 \\
\hline
\end{tabular}


Table 2. Cont.

\begin{tabular}{|c|c|c|c|c|c|c|c|c|}
\hline \multirow{2}{*}{ Rt (min). } & \multirow{2}{*}{ MS; MS2 ( $m / z)$} & \multirow{2}{*}{ Anthocyanin } & \multicolumn{2}{|c|}{ V. amurensis } & \multicolumn{2}{|c|}{ Interspecific Hybrids } & \multicolumn{2}{|c|}{ V. vinifera } \\
\hline & & & Mean & SE & Mean & SE & Mean & SE \\
\hline 6.6 & $817 ; 655,493,331$ & Mv-3-cfglc-5-glc & 2.24 & 0.00 & 21.62 & 32.73 & - & - \\
\hline 6.56 & $611 ; 287$ & Cy-3-cfglc & - & - & 0.07 & 0.04 & 0.56 & 0.91 \\
\hline 6.61 & $757 ; 595,449,287$ & Cy-3-cmglc-5-glc & 1.07 & 1.49 & 23.54 & 35.97 & - & - \\
\hline 6.62 & $\begin{array}{c}787 ; 625,479,317 \\
/ 787 ; 625,463,301\end{array}$ & $\begin{array}{l}\text { Pt-3-cmglc-5-glc } \\
\text { /Pn-3-cfglc-5-glc }\end{array}$ & 4.24 & 8.92 & 54.50 & 107.03 & - & - \\
\hline 6.84 & $505 ; 301$ & Pn-3-acglc & 0.36 & 0.33 & 3.07 & 3.19 & 29.84 & 21.99 \\
\hline 6.91 & $535 ; 331$ & Mv-3-acglc & 1.83 & 1.68 & 22.46 & 29.21 & 191.51 & 38.03 \\
\hline 6.73 & $641 ; 317$ & Pt-3-cfglc & - & - & 0.25 & 0.29 & 0.46 & 0.60 \\
\hline 6.93 & $611 ; 303$ & Dp-3-cmglc & 0.51 & 0.56 & 2.25 & 4.85 & 8.49 & 14.50 \\
\hline 7.07 & $771 ; 609,463,301$ & Pn-3-cmglc-5-glc & 0.94 & 1.06 & 14.56 & 10.38 & - & - \\
\hline 7.02 & $801 ; 639,493,331$ & Mv-3-cmglc-5-glc & 1.10 & 1.08 & 94.32 & 142.02 & - & - \\
\hline 7.07 & $625 ; 301$ & Pn-3-cfglc & - & - & 0.19 & 0.23 & 4.53 & 6.12 \\
\hline 7.23 & $655 ; 331$ & Mv-3-cfglc & - & - & 0.57 & 1.50 & 18.88 & 15.31 \\
\hline 7.37 & $595 ; 287$ & Cy-3-cmglc & - & - & 0.13 & 0.24 & 3.62 & 6.06 \\
\hline 7.48 & $625 ; 317$ & Pt-3-cmglc & - & - & 0.69 & 1.18 & 14.31 & 23.56 \\
\hline 8.25 & $609 ; 301$ & Pn-3-cmglc & - & - & 0.36 & 0.69 & 29.73 & 42.93 \\
\hline 8.37 & $639 ; 331$ & Mv-3-cmglc & 0.00 & 0.00 & 4.60 & 10.86 & 197.95 & 256.04 \\
\hline
\end{tabular}

* indicates the anthocyanin compound was speculated according to $m / z$ values. And they were quantified using malvidin-3,5-O-diglucoside as standards. \# indicates the two anthocyanin compounds were not separated according to $m / z$ values.And they were quantified as the $p$-coumaroyl derivatives due to the fragment ion abundance. Abbreviations: $\mathrm{Dp}$, delphinidin; $\mathrm{Cy}$, cyanidin; Pt, petunidin; Pg, pelargonidin; Pn, peonidin; Mv, malvidin; triglc, triglucosides; diglc, diglucosides; glc, monoglucoside; acglc, (6-acetyl)-glucoside; cmglc, (6-coumaroyl)-glucoside; cfglc, (6-caffeoyl)-glucoside.

A new class of anthocyanins, eluted at the earliest (2-4 min, Table 2), was detected in all the $V$. amurensis grapes and hybrids, but not in the three $V$. vinifera grapes. These molecular ions $[\mathrm{M}]^{+}$were $789,773,803,787$ and 817 in chronological order, and their mass spectrum were characterized by the three fragment ions of $[\mathrm{M}-162]^{+},[\mathrm{M}-324]^{+}$and $[\mathrm{M}-486]^{+}$caused by the stepwise cleavage of three glucose units (Figure 3a). In the chemical structures of anthocyanidins there are three phenolic hydroxyl groups at the positions C 3 , C5 and $\mathrm{C7}$, respectively. In grapes, the monoglucoside anthocyanin (3-O-glucoside) is produced by the glucosylation of one hydroxyl group at $\mathrm{C} 3$, while the diglucosides anthocyanin (3,5-O-diglucosides) is produced by the glucosylation of the two hydroxyl groups at C3 and C5, respectively. Although 3,7-diglucosides of anthocyanidins, shown to exist in some flowers [33], have been detected in red wine, this was not entirely certain [34]. These compounds should not be the caffeoyl diglucosides anthocyanins, which possess similar molecular ions and are eluted behind all the simple glucoside anthocyanins and acetyl diglucosides anthocyanins. So, we preliminarily speculate that these new anthocyanins were the 3,5,7-O-triglucosides of $\mathrm{Dp}, \mathrm{Cy}, \mathrm{Pt}, \mathrm{Pn}$ and $\mathrm{Mv}$, respectively. In addition, a $p$-coumaroyl triglucoside anthocyanin $\left([\mathrm{M}]^{+}, 963\right)$ was identified in all the interspecific hybrids except for 'Zuohong-1' and 'Zuoyouhong', and was assigned to Mv-3-O-(6-O$p$-coumaryl)-5-O-7-O-triglucosides by the loss of two glucosides $(\mathrm{m} / z \mathrm{z01}, 639)$ and a coumaroyl glucoside $(m / z 331)$ (Figure $3 b)$. To our knowledge, this is the first time the detection of triglucoside anthocyanins in grapes has been reported. However, further research is still needed to confirm this speculation. 

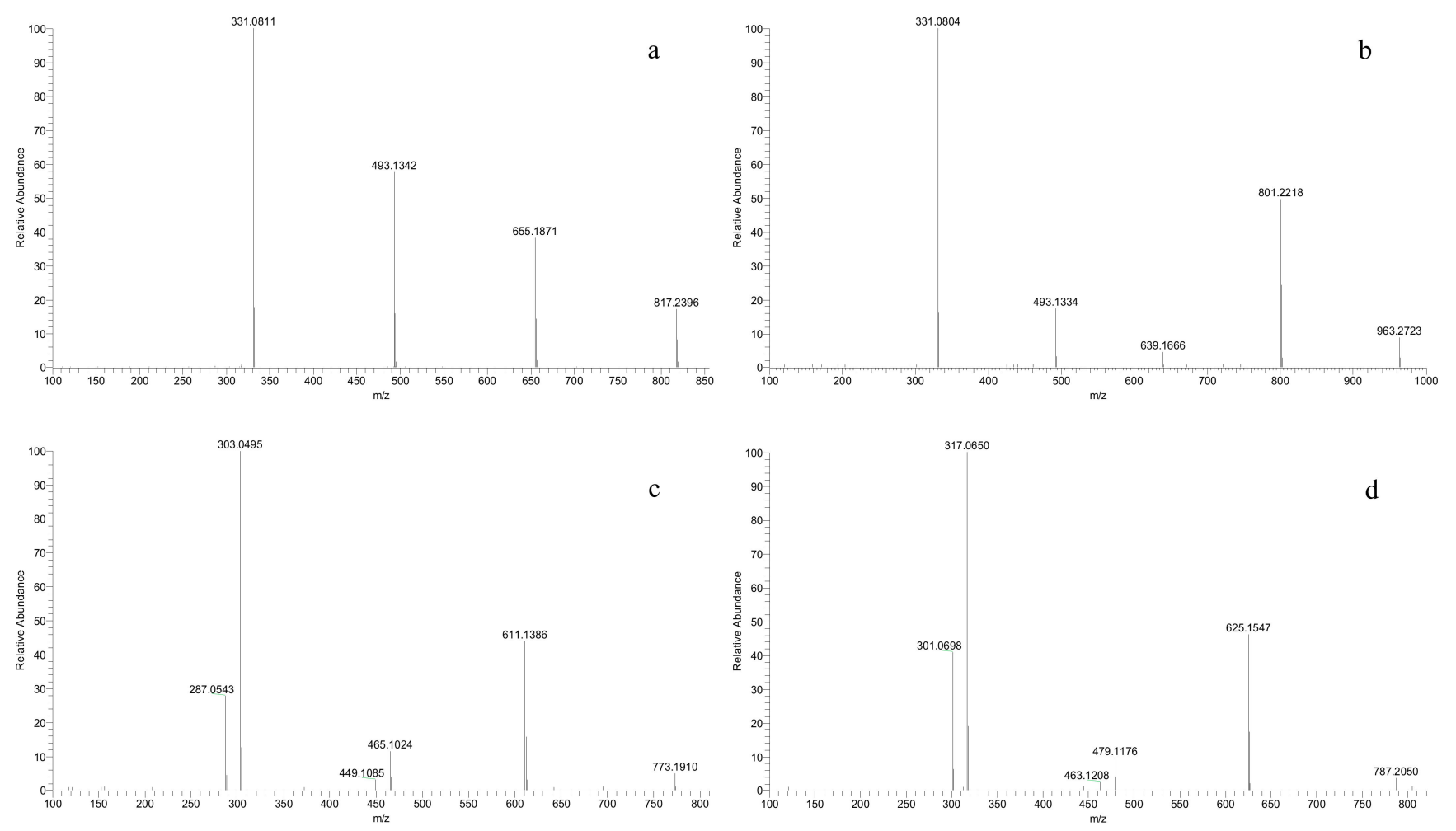

Figure 3. $\mathrm{MS}^{2}$ spectrum of the ion signals $m / z 817(\mathbf{a}), 963(\mathbf{b}), 773$ (c) and 787 (d).

Under the condition of separation and detection in this study, two couples of anthocyanins with the same molecular ions $[\mathrm{M}]^{+}$were eluted almost at the same time. In other words, multiple fragment ions contributed to the final intensity of a molecular ion signal in the mass spectrum. For example, Dp-3-O-(6-O-p-coumaryl)-5-O-diglucoside $\left([\mathrm{M}]^{+}, 773\right)$ was indistinguishable from $\mathrm{Cy}-3-\mathrm{O}-(6-\mathrm{O}$-caffeoyl)-5-O-diglucoside (Table 2, Figure 3c). Similarly, Pt-3-O-(6-O-p-coumaryl)-5-O-diglucoside ([M] $\left.{ }^{+}, 787\right)$ was isobaric with Pn-3-O(6-O-caffeoyl)-5-O-diglucoside (Table 2, Figure $3 \mathrm{~d}$ ). However, the fragment ion abundance of $p$-coumaroylation was obviously stronger than that of caffeoylation, which indicated that caffeoyl derivatives were few and insignificant.

\subsubsection{Total Anthocyanin Contents}

The total anthocyanin contents (TA) varied significantly among different grape groups (Figure 4a, Table S1). The $V$. amurensis grapes had the highest average TA (31.83 mg MGE/g DM). The V. amurensis accession ' $086919^{\prime}$ ' had the most TA (45.13 mg MGE/g DM) among all the grapes assayed, followed by ' $85,010^{\prime}$ ', '92', ' 14 ', 'Changbai-5', 'Zuoshan-1' and 'Zuoshan$2^{\prime}$, with more than $40 \mathrm{mg}$ MGE/g DM. The lowest average TA (3.60 mg MGE/g DM) was found in the three $V$. vinifera cultivars, and the hybrids' contents (10.07 mg MGE/g DM) fell between $V$. amurensis and $V$. vinifera. 

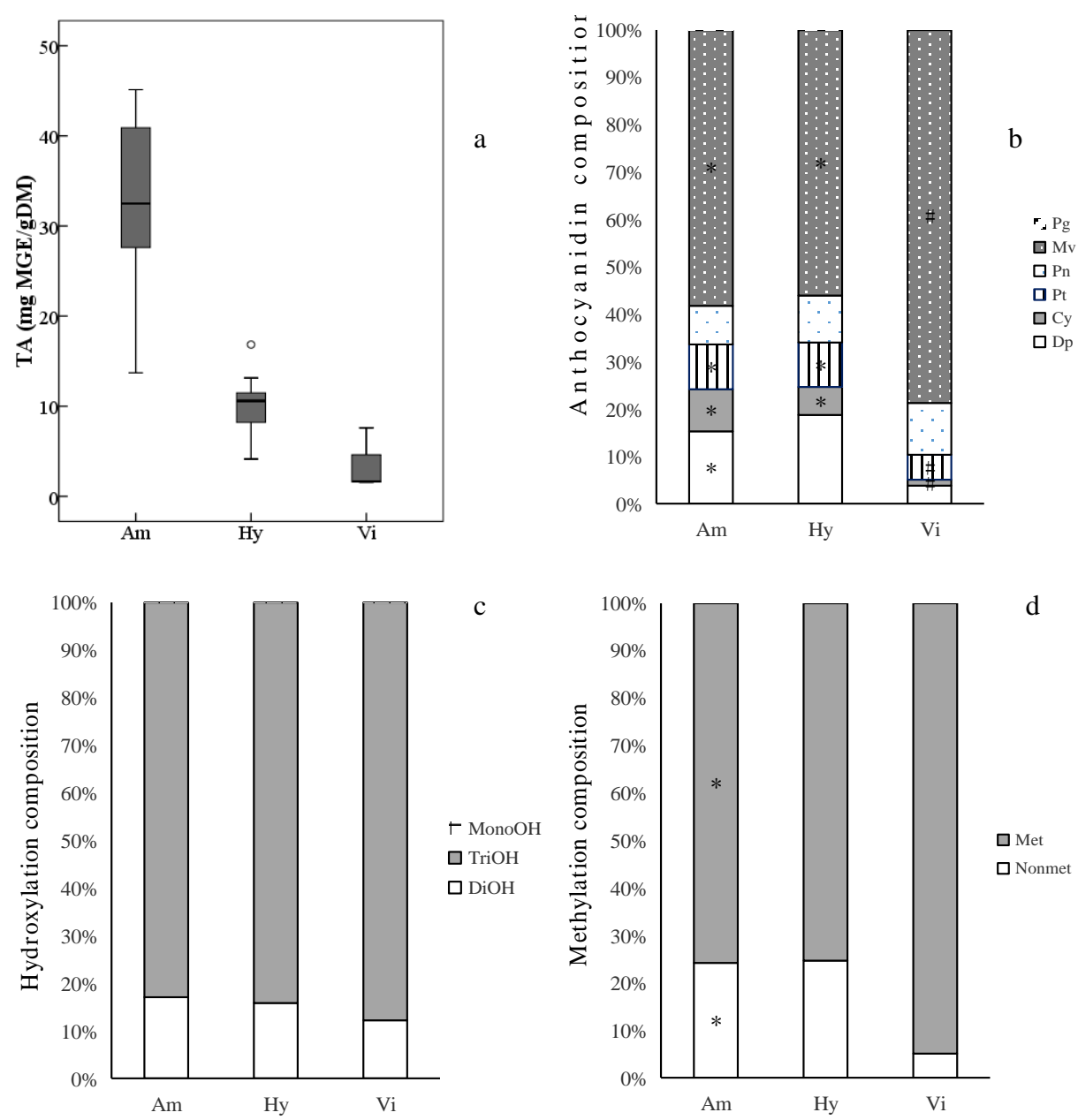

d
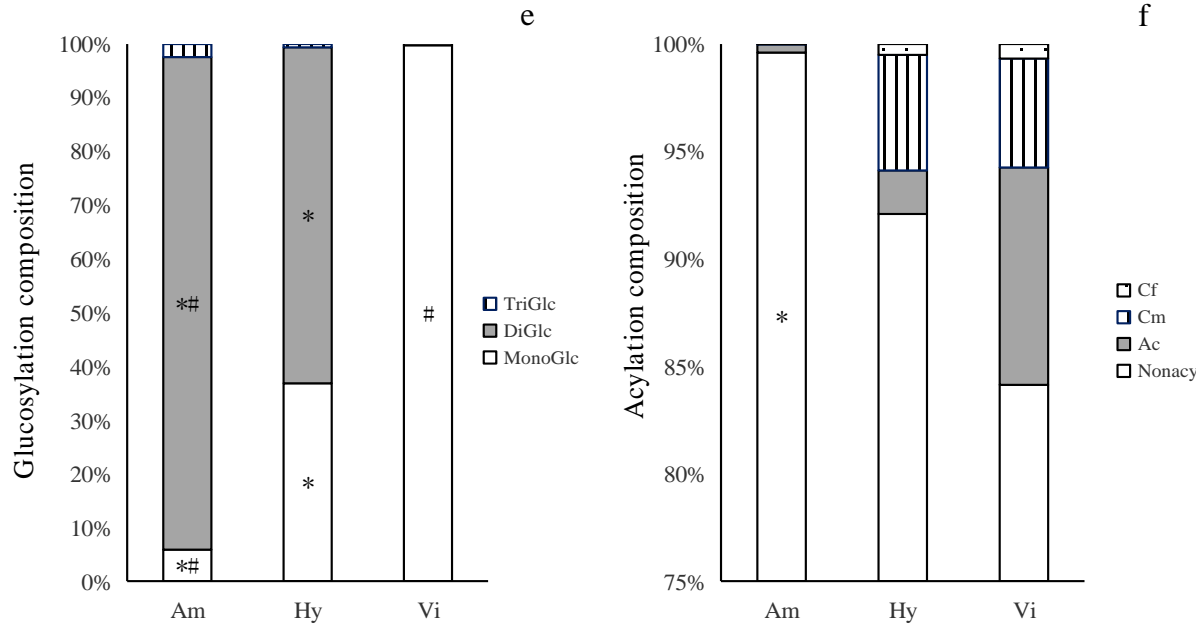

Figure 4. Ranges and distributions of total anthocyanins (TA, a) and anthocyanin compositionsof anthocyanidin (b), hydroxylation (c), methylation (d), glucosylation (e) and acylation (f) in ripe berry skins belonging to the three grape groups from 2017 2018. * indicates significant difference $(p<0.05)$ vs. V. vinifera grapes for each indicator by Welch's ANOVA with the Games-Howell test. \# indicates significant difference $(p<0.05)$ vs. interspecific hybrids. Abbreviations: Dp, delphinidin derivatives; $\mathrm{Cy}$, cyanidin derivatives; $\mathrm{Pt}$, petunidin derivatives; $\mathrm{Pg}$, pelargonidin derivatives; $\mathrm{Pn}$, peonidin derivatives; $\mathrm{Mv}$, malvidin derivatives; $\mathrm{MonoOH}$, monohydroxylated anthocyanins; $\mathrm{DiOH}$, dihydroxylated anthocyanins; TriOH, trihydroxylated anthocyanins; Met, methylated anthocyanins; Nonmet, nonmethylated anthocyanins; MonoGlc, monoglucoside anthocyanins; DiGlc, diglucosides anthocyanins; TriGlc, speculated triglucosides anthocyanins; Cf, caffeoyl anthocyanins; Cm, coumaryl anthocyanins; Ac, acetyl anthocyanins; Nonacy, nonacylated anthocyanins. The abbreviations of grape groups follow Figure 1. 


\subsubsection{Composition of Anthocyanins}

Among the derivatives of different anthocyanidins, Mv-derivatives were the most abundant components in the three grape groups, which was in agreement with our previous research on V. vinifera cultivars [35] and East Asian species [18].The average percentages of Mv-derivatives in the skins of $V$. amurensis grapes (58\%) and hybrids (56\%) were significantly less than that in the three $V$. vinifera grape skins (80\%) (Figure $4 \mathrm{~b}$ ), and was approximate for the average percentages of Pn-derivatives among the $V$. vinifera grapes $(10 \%), V$. amurensis grapes $(8 \%)$ and hybrids $(10 \%)$, while the average percentages of Dpderivatives, Cy-derivatives and Pt-derivatives in V. amurensis grapes $(15 \%, 9 \%$ and $9 \%)$ and hybrids $(19 \%, 6 \%$ and $9 \%)$ were obviously higher than those in the three $V$. vinifera grapes $(4 \%, 1 \%$ and $5 \%)$. In addition, it has been confirmed that Pg-derivatives are present at trace concentrations in some grape cultivars of $V$. vinifera [36,37], V. amurensis [10,21], V. labrusca, V. aestivalis [18] and V. rotundifolia [38]. In this study, pg-derivatives were found in all the grapes assayed, accounting for $0.00046-0.10 \%$ of TA (Figure $4 \mathrm{~b}$ ). Only two simple glucoside derivatives, $\mathrm{Pg}$-3-O-glucoside and Pg-3,5-O-diglucosides, were detected (Table 2). However, there was a significant difference in the anthocyanidin compositions among the different grapes. For example, the interspecific hybrids 'Hasang' and 'Zuohong-1', as well as the $V$. amurensis accession 'Tonghua- 1 ', had significantly smaller proportions of Mv-derivatives (2\%, 26\% and $12 \%$ ) than the other grapes, while 'Hasang' had the highest percentages of Dp-derivatives (83\%) among all the grapes assayed, followed by 'Tonghua-1' (55\%) and 'Zuohong- 1 ' (45\%).

The six kinds of anthocyanidin derivatives can be divided according to the positions and numbers of the hydroxyl and methoxyl substituents in their B rings [39]. The degree of anthocyanins' hydroxylation affects the hue and stability of their colors. The more hydroxyl groups in their B rings, the more blue anthocyanins appear [39]. In this study, the trihydroxylated anthocyanins were the richest in all the grape skins collected. There was no difference the average percentages of trihydroxylated anthocyanins among the $V$. vinifera grapes $(88 \%), V$. amurensis grapes (83\%) and interspecific hybrids (84\%) (Figure 4c). More methoxyl groups in anthocyanins' B rings contribute more redness [40], and methylated derivatives (Pt-, Pn- and Mv-derivatives) were dominant in most grapes (Figure 4d) except in 'Hasang', 'Zuohong-1' and 'Tonghua- 1 '. The average percentages of methylated derivatives were lower in V. amurensis grapes $(76 \%)$ and hybrids $(75 \%)$ compared with the three $V$ vinifera grapes (95\%) (Figure 4c).

There was a distinct separation in the glucosylated composition of the anthocyanins of the different grape groups (Figure 4e). The diglucoside anthocyanins were predominant in the $V$. amurensis grapes (91.71\% of TA on average), which was consistent with the results of previous studies $[18,19,21]$. The average percentage of diglucoside anthocyanins was significantly less in the hybrids of V. amurensis and V. vinifera $(63 \%)$, but there was an obvious difference in glucosylated composition among the interspecific hybrids. The percentage of diglucosides anthocyanins ranged from 33\%, in 'Zuohong-1', to $86 \%$, in 'RS'. In the three $V$. vinifera wine grapes, almost all of anthocyanins were monoglucoside derivatives. In addition, the speculated triglucoside anthocyanins found in V. amurensis grapes and hybrids were present only in trace amounts.

Razgonova et al. [10] identified 7 acylated derivatives, out of a total of 18 anthocyanin compounds, in V. amurensis grapes from Far East Russia. In this study, a richer group of acylated anthocyanin compounds (31) were detected (Table 2). However, it has been reported that $V$. amurensis grapes snone [18,21] or a few [41] acylated anthocyanins. A similar result, of only $0.40 \%$ content acylated anthocyanins of TA in all the $V$. amurensis grapes on average (Figure $4 \mathrm{f})$, was obtained in this study. In $V$. amurensis grapes, acetyl anthocyanins $(0.38 \%)$ were the main and common acylated type; only trace coumaryl anthocyanins were found. In the interspecific hybrids, acetyl, coumaryl and caffeoyl anthocyanins accounted for $2 \%$, $5 \%$ and $0.05 \%$ of TA on average and respectively, but acylation varied widely, due to the samples' different pedigrees. The contents and compositions of acylated anthocyanins in 'Zuohong- 1 ' and 'Zuoyouhong' were extremely similar with $V$. amurensis grapes. An 
outstanding exception was 'Hasang', with the highest percentage of acylated anthocyanins (51\%) among all the assayed grapes, while 'Beichun' (9\%), 'Huapu-1' (9\%) and 'RS' (7\%) also had relatively higher percentage of acylated anthocyanins. 'Beichun' had the highest percentage of acetyl anthocyanins $(7 \%)$ among all the hybrids, while coumaryl were significantly more than acylated anthocyanins in 'Hasang', 'Huapu-1', 'RS' and 'Gongniang-1'. In the three $V$. vinifera grapes, the acylated anthocyanins accounted for higher proportions $(16 \%)$ than those in $V$. amurensis grapes and hybrids on average, which was similar to the results of Liang et al. [35,41].

\subsection{Antioxidant Properties of Phenolic Extracts from Grape Skins and Seeds}

In this study, the antioxidant capacities (Figure 2d-f, Table S1), in vitro, were assayed by DPPH, ABTS and FRAP. For the seeds, the variation of the average antioxidant values among grape groups showed a similar tendency with respect to total contents of phenolics. The average values of antioxidant indicators were significantly lower in the $V$. amurensis grapes and hybrids than in the $V$. vinifera grapes. For the skins, the average level of antioxidant activities decreased in the order of $\mathrm{Vi}>\mathrm{Am}>\mathrm{Hy}$, which differed from the distribution of total phenolic contents; but the difference of antioxidant values was not significant between $V$. vinifera and $V$. amurensis.

\subsection{Correlation Analysis}

The total phenolic contents in the seeds presented a more obvious consistency as compared with the skins, which maybe because the seed phenolics mainly consisted of flavan-3-ols [30], while the skin phenolics contained more variety, such as phenolic acids, flavonols, anthocyanins and flavan-3-ols [18]. This was confirmed by the correlation analysis among the total phenolic contents. There were extremely high correlations in the seeds (0.997-0.999, Table S3), while in the skins, the correlations were positive and significant among TP, TFO and TA (0.644-0.963) and TFA had low correlations with TP (0.036) and TFO (0.060) and a negative correlation with TA (-0.500, Table S2). It was indicated that the main phenolic type was anthocyanins rather than flavan-3-ols (mainly tannins) in the skins of red wine grapes collected in this study, especially $V$. amurensis.

Correlation analysis was also performed between samples' phenolic contents and antioxidant activities. Generally, the richer the phenolic content grape materials possess, the stronger the antioxidant activities their phenolic extracts $[30,42,43]$. The correlations in the grape seeds were extremely significant (0.965-0.989, Table S3), and there were also significant correlations (0.257-0.896, Table S2) except for the coefficients of TFA and FRAP (0.197). The differences between the seeds and skins should be due to the complex composition of phenolic compounds in skins and the low flavan-3-ols in V. amurensis grape skins. Among the anthocyanin compounds, only the simple diglucoside derivatives, the most abundant type in $V$. amurensis grapes, significantly contributed to the antioxidant capacities of the skin phenolic extracts $(0.237-0.471$, Table S4).

\subsection{Principle Component Analysis}

In order to separate these grape cultivars/accessions and describe the phenolic characteristics of different grape groups, a principal component analysis (PCA) was carried out on the basis of anthocyanin composition, total contents and the antioxidant activities of their phenolic compounds. A total of six principal components with eigenvalues $>1$ were obtained, explaining $91.69 \%$ of total variance. PC1 and PC2 possessed relatively high percentages of variance ( $36.32 \%$ and $20.06 \%$, respectively). PC1 was mainly represented by total contents and antioxidant activities of seed phenolic compounds, skin TFA and the percentage of acetylated anthocyanins with positive correlations, as well as TA, TP, TFO and the percentages of diglucosides anthocyanins and Cy-derivativesin skins with negative correlations. PC2 consisted primarily of the antioxidant activities of the skins ${ }^{\prime}$ phenolics and the percentage of Dp-derivatives, coumaryl and caffeoyl anthocyanins with 
positive $\mathrm{PC} 2$ values, as well as nonacylated and methylated anthocyanins with negative PC2 values (Figure 5a).

A scatter plot on the basis of PC1 and PC2 (Figure $5 b$ ) showed that the cultivars/accessions from the same group appeared to occupy a certain PC1 range. The V. amurensis cultivars/accessions, located on the negative side of the PC1 axis, were characterized by having high TA and proportions of diglucoside anthocyanins. The three $V$. vinifera grapes were located on the far positive side of the PC1 axis, mainly because of their high total contents and the antioxidant capacities of the phenolic compounds in their seeds, as well as the high TFA and proportions of acetylated anthocyanins in their skins. As expected, the hybrid grapes were located between the $V$. amurensis and $V$. vinifera grapes on the $\mathrm{PC} 1$ axis, with a value range of $-0.53-1.20$. There was an exception - the hybrids 'Hasang' had an extremely high PC2 values (4.25) compared with other grapes (-1.41-1.53), due to its high proportions of Dp-derivatives, coumaryl and caffeoyl anthocyanins.
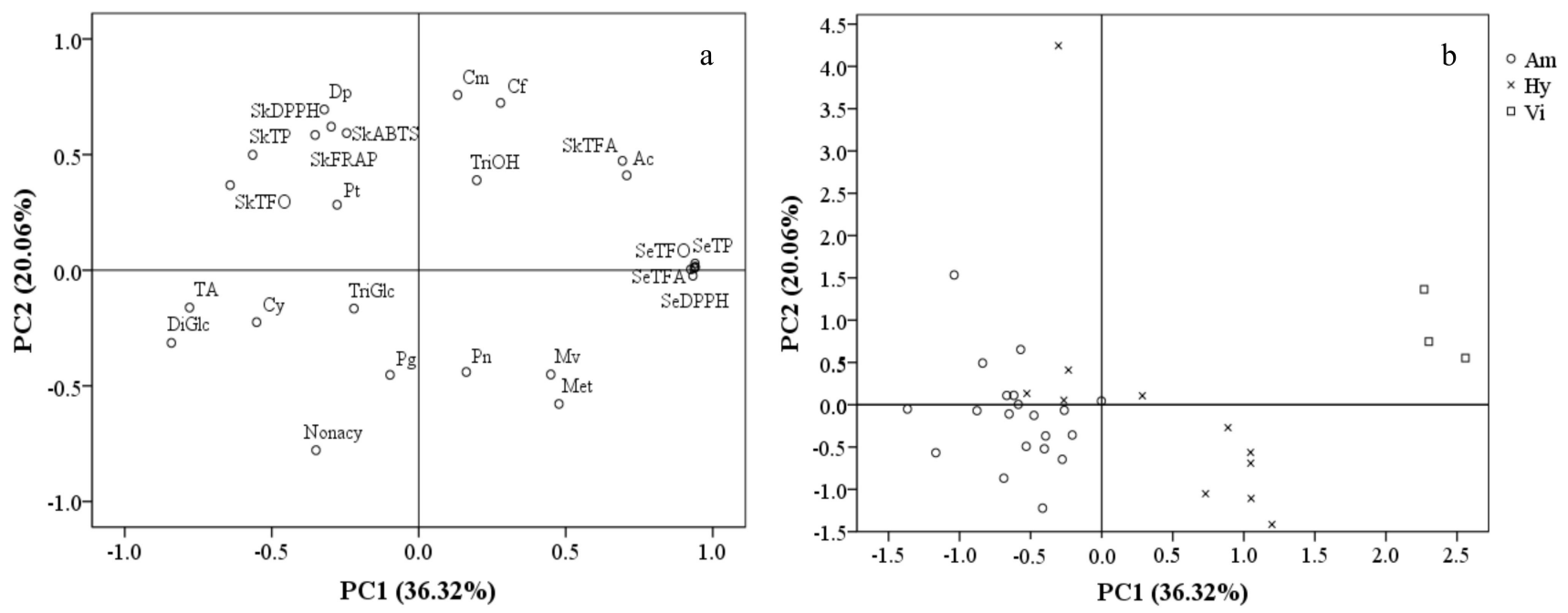

Figure 5. Distribution patterns of 27 variables (a) and 34 cultivars/accessions belonging to the three grape groups (b) 2017 and 2018 in the two-dimensional space of PC1 and PC2. The abbreviations of variable and grape groups follow Figures 1, 2 and 4.

\section{Discussion}

\subsection{Selection of Core V. amurensis Germplasm}

There are abundant $V$. amurensis germplasm resources in China. Through domestication and selection of wild resources, a number of $V$. amurensis cultivars have been screened out. In 1957, the female flower lines of 'Changbai' and 'Tonghua' were selected, outstandingly represented by 'Changbai-9' and 'Tonghua-3'. It was a very exciting discovery that 'Changbai-11' was a hermaphrodite flower cultivar, and officially named as 'Shuangqing' in 1975. It was the first ever finding of a V. amurensis resource with hermaphrodite flowers, which ended the commercial production of female flower cultivars with the male pollenizers [44]. Other female flower cultivars, 'Zuoshan-1' and 'Zuoshan-2', were successfully selected in the middle of the 1980s. Based on the wild V. amurensis, a series of intraspecific hybrids with bisexual flowers were bred from the female parents of pistillate flower genotypes and a male parent of 'Shuangqing', such as 'Shuangfeng' [45], 'Shuangyou' [46] and 'Shuanghong' [47]. Through the crossing between V. amurensis and V. vinifera, plenty of new interspecific hybrids were selected from the filial generations, including the red wine cultivars 'Zuohong-1' [48], 'Zuoyouhong' [49], 'Xuelanhong' [50], 'Beichun' [51], 'Beihong', 'Beimei' [52], 'Gongniang-1', 'Gongniang-2' [53] and 'Huapu-1' [54], as well as the ice wine cultivar 'Beibinghong' [55]. Compared with V. amurensis grapes, the interspecific hybrids had better qualities for making wine, such as relatively high sugar and low acid [5]. These hybrids considerably enhanced production and promoted the industry's development of $V$. amurensis [5]. In this study, the above representative V. amurensis cultivars and hybrids 
and important accessions from different regions were collected from the VAGR of CAAS to investigate the quality characteristics and anthocyanin profiles of the overall $V$. amurensis germplasm in China.

\subsection{General Characteristics and Total Phenolic Contents of V. amurensis Grapes and Hybrids}

The mature berries of $V$. amurensis collected in this study were round and small, which matched the descriptions of Liu and Li [5] and Chen et al. [6]. Among the 20 studied $V$. amurensis grapes, their transverse diameters were only $0.03 \mathrm{~mm}$ (Table S1) smaller than their longitudinal diameters, on average. However, the obtained range of $9.84-13.15 \mathrm{~mm}$ of the diameters in this study was longer than that of 5-12 mm, measured by Zhuang [56]. Our average berry weights (1.02 g, Table S1) were heavier than the $10 \mathrm{~V}$. amurensis accessions $(0.83 \mathrm{~g})$ studied by Liang et al. [41]. V. amurensis grapes have beenproven to produce high acid and relatively low sugar [5]. In this study, the total soluble solids were 9.56 Brix to 17.22 Brix, which was similar to the sugar content range $(80-170 \mathrm{~g} / \mathrm{L})$ concluded by Jie [57]. The observed titratable acids ranged from $0.67 \%$ to $2.04 \%$, which were lower than the organic acid contents (15-30 g/L) concluded by Jie [57], mainly because of the relatively lower acid content of six $V$. amurensis cultivars, 'Zuoshan-1', 'Zuoshan-2', 'Shuangqing', 'Shuangyou', 'Shuanghong' and 'Shuangfeng', that fall a range of $0.67-1.27 \%$. The differences in the general characteristics were perhaps induced by the various genotypes and terroir.

The distribution of phenolic contents between seeds and skins were different between $V$. amurensis and $V$. vinifera. The TP and TFO contents in skins were higher than in seeds for most $V$. amurensis cultivars/accessions, with a few exceptions; the $V$. vinifera grapes had much higher total phenolic contents in their seeds than in their skins, which agrees with that found in other studies $[42,58,59]$. The performances among interspecific hybrids were different due to various breeding parents, butfor all the grapes assayed, TFA was much richer in seeds than in skins. In general, the $V$. amurensis grapes collected in this study had richer phenolic compounds in their skins, except for flavan-3-ols, but lower phenolic contents in their seeds compared with $V$. vinifera grapes.

\subsection{Anthocyanin Profiles of V. amurensis Grapes and Hybrids}

There is abundant anthocyanin content in $V$. amurensis. In this study, the average TA in $V$. amurensis grape skins was 8.18-fold higher than that in the skins of the three $V$. vinifera wine grapes. In previous studies, the levels of TA in entire $V$. amurensis samples was comparatively high compared with $V$. vinifera $[35,41]$, other East Asian species [18] and American species [19,41].

A proportion of more than $90 \%$ diglucoside anthocyanins is the most important trait in the anthocyanin composition of $V$. amurensis. Diglucoside anthocyanins are more stable, compared with monoglucoside anthocyanins, due to their molecular structures, but diglucoside anthocyanins in wines form stable and complex pigments with relative difficulty, compared with monoglucoside anthocyanins, such that aged wines with a certain amount of diglucoside anthocyanins are more susceptible to browning and retain less color $[39,60]$. In addition, a significantly smaller proportion of acylated anthocyanins was found in $V$. amurensis compared with $V$. vinifera. Acylation may increase the stability and solubility of anthocyanin molecules [39]. As a result, V. amurensis grapes and hybrids are poorly suited to the production of aged red wines. Together with high acids and low sugar, $V$. amurensis grapes and hybrids are mainly suitable for sweet wines. Thus, grape breeders in China have been devoted to improving the wine-making quality of $V$. amurensis by the crossbreeding it with $V$. vinifera.

Grapes possess a rich variety of anthocyanin compounds because of the functions of modification enzymes in anthocyanin biosynthesis, such as hydroxylases, methyltransferases, glucosyltransferases and acyltransferase. The compounds 5-O-glucosyltransferases (5GT) are responsible for converting monoglucoside anthocyanins into diglucoside anthocyanins. It was reported for the first time that the nonfunctional 5GT allele in the 
No. 9 chromosome (chr 9) might result in the inability of $V$. vinifera grapes to synthesize diglucoside anthocyanins [61]. Through the cloning and analysis of the 5GT allele in chr 9 from $V$. vinifera and other 7 Vitis spcies, Yang et al. [43] found that 27 functional 5GT alleles were present in non- $V$. vinifera Vitis species, and 18 out of $265 \mathrm{GT}$ alleles were apparently nonfunctional in $V$. vinifera. He et al. [62] isolated successfully a $5 G T$ allele in chr 9 from $V$. amurensis 'Zuoshan- 1 ', whose recombinant proteins were confirmed to have a 5GT function by enzymology tests; and a functional $5 G T$ allele was found in chr 5 of $V$. vinifera 'Cabernet Sauvignon' [63], which might explain the trace diglucosides anthocyanins $(0.23 \%$ on everage) detected in the three $V$. vinifera grapes of this study. However, the biosynthesis and regulation mechanisms of diglucoside anthocyanins need to be explored father, as it could provide a theoretical basis for the reduced proportion of diglucoside anthocyanins in V. amurensis.

It is interesting that a new class of anthocyanins was found in all the examined $V$. amurensis grapes and hybrids. According to its mass spectra, it is preliminarily speculated that these anthocyanins are the 3,5,7-O-triglucosides of five anthocyanidins (Dp, Cy, Pt, Pn and $\mathrm{Mv}$ ). Also, a $p$-coumaroyl triglucosides of $\mathrm{Mv}$ was detected in most of the interspecific hybrids. To our knowledge, this is the first time the detection of triglucosides anthocyanins in grapes has been reported, and the above-mentioned new anthocyanin type has not been found in other plant species. The speculated triglucoside anthocyanins might be the analytic targets for identifying grapes and wines with the $V$. amurensis pedigree, but further research is still needed to confirm this speculation.

\section{Conclusions}

In this study, we investigated and analyzed the berry qualities of important and representative $V$. amurensis grapes and interspecific hybrids maintained in the VAGR of CAAS. Three red wine grapes of $V$. vinifera, widely cultivated throughout the world, were also collected as a comparative group. The survey items included general characteristics, total contents and antioxidant activities of phenolic compounds, and especially of anthocyanin contents and compositions. In general, the anthocyanins of $V$. amurensis grapes featured rich total contents, high diglucosylation and low acylation levels. With the application of UPLC/Q-Exactive orbitrap MS, a total of 48 anthocyanins were detected and a new type of speculated 3,5,7-O-triglucoside anthocyanins were identified in grapes with the $V$. amurensis pedigree. In addition, the ripe $V$. amurensis berries had higher contents of titratable acids, total phenols and flavonoids in skins, and lower total soluble solids, phenolics in seeds, flanan-3-ols and antioxidant activities. Through correlation analysis, the contents of total phenolic compounds (except for flanan-3-ols in skins) and simple diglucosides anthocyanins obviously contributed to the antioxidant activities of phenolic extracts. Most characteristics of the interspecific hybrids fell between those of $V$. amurensis and $V$. vinifera, which indicated interspecific breeding with $V$. vinifera could improve the wine-making quality of $V$. amurensis. This work is an important component in our ongoing effort to develop a comprehensive database the qualities and nutritional characteristics of $V$. amurensis germplasms for breeding and commercialization.

Supplementary Materials: The following are available online, Table S1. General berry characteristics, total contents and antioxidant activities of phenolic compounds extracted from grape skins and seeds in the three groups during the 2017 and 2018 years; Table S2. Correlation coefficients among total phenolic contents and antioxidant activities of phenolic compounds in grape skins. Table S3. Correlation coefficients among total phenolic contents and antioxidant activities of phenolic compounds in grape seeds. Table S4. Correlation coefficients among the contents of simple diglucosides anthocyanins and antioxidant activities of phenolic extracts in grape skins.

Author Contributions: Conceptualization and writing-original draft preparation, L.Z.; methodology, L.Z., X.L. and X.W.; formal analysis, Y.L. and H.T.; resources and investigation, X.H. and Y.Y.; figures, Y.Z.; writing-review and editing, J.X. and C.W. All authors have read and agreed to the published version of the manuscript. 
Funding: This research was funded by Natural Science Foundation of Heilongjiang Province (Grant No. QC2017024), National Key Research and Development Program of China (Grant No. SQ2018YFE020070 and 2018YFE0206300) and Platform Support Program for "San Zong San Heng" of Heilongjiang Bayi Agricultural University (Grant No. PTJH201904).

Institutional Review Board Statement: Not applicable.

Informed Consent Statement: Not applicable.

Data Availability Statement: The data presented in this study are available in Supplementary Materials.

Acknowledgments: We are grateful to Jiang Lu at Shanghai Jiao Tong University, Jun Wang at China Agricultural University and Jun Ai at Jilin Agricultural University for their assistance in collecting berry samples. The UPLC-MS determination was performed in the Testing Centre of Qiqihar University, and we also thank Zhe Wang and Yan Jiao at Qiqihar University for their assistance in the determination work.

Conflicts of Interest: The authors declare no conflict of interest.

Sample Availability: Samples of the compounds are not available from the authors.

\section{References}

1. Shen, Y.J.; Zhao, S.L.; Yang, Y.M.; Li, X.H.; Song, R.G.; Lu, W.P. The research and utilization on Amur grape (V. amurensis Rupr.) germplasm resources in China. Spec. Wild Econ. Anim. Plant Res. 2006, 28, 53-57.

2. Zhang, Q.T.; Lu, W.P.; Fan, S.T.; Yang, Y.M.; Ai, J.; Song, R.G. Research progress and prospect of Vitis amurensis Rupr. breeding in China. J. Heibei For. Sci. Technol. 2014, 5, 121-123.

3. He, P.C.; Wang, Y.J.; Wang, G.Y.; Pen, Z.B.; He, C.C. The studies on the disease-resistance of Vitis wild species originated in China. Sci. Agric. Sin. 1991, 24, 50-56.

4. Song, R.G.; Ai, J.; Li, X.H.; Yan, Y.M.; Shen, Y.J. Industrial development and countermeasure V. amurensis Rupr. in China. Sino-Overseas Grapevine Wine 2009, 11, 64-69.

5. Liu, L.Y.; Li, H. Review: Research progress in amur grape, Vitis amurensis Rupr. Can. J. Plant Sci. 2013, 93, 565-575. [CrossRef]

6. Chen, Q.; Li, C.; Song, H.; Zhu, X. Vitis amurensis Rupr: A review of chemistry and pharmacology. Phytomed. Int. J. Phytother. Phytopharm. 2018, 49, 111-122. [CrossRef] [PubMed]

7. Hilbert, G.; Temsamani, H.; Bordenave, L.; Pedrot, E.; Chaher, N.; Cluzet, S.; Richard, T. Flavonol profiles in berries of wild Vitis accessions using liquid chromatography coupled to mass spectrometry and nuclear magnetic resonance spectrometry. Food Chem. 2015, 169, 49-58. [CrossRef]

8. Ji, M.; Li, C.; Li, Q. Rapid separation and identification of phenolics in crude red grape skin extracts by high performance liquid chromatography coupled to diode array detection and tandem mass spectrometry. J. Chromatogr. A 2015, 1414, 138-146. [CrossRef] [PubMed]

9. Zhao, Q.; Duan, C.Q.; Wang, J. Components of non-anthocyanin phenolic compounds in wines of Vitis amurensis and its hybrids. Afr. J. Biotechnol. 2011, 10, 14767-14777. [CrossRef]

10. Razgonova, M.; Zakharenko, A.; Pikula, K.; Manakov, Y.; Ercisli, S.; Derbush, I.; Kislin, E.; Seryodkin, I.; Sabitov, A.; Kalenik, T.; et al. LC-MS/MS screening of phenolic compounds in wild and cultivated grapes Vitis amurensis Rupr. Molecules 2021, 26, 3650. [CrossRef] [PubMed]

11. Min, Z.; Guo, Z.; Wang, K.; Zhang, A.; Li, H.; Fang, Y. Antioxidant effects of grape vine cane extracts from different Chinese grape varieties on edible oils. Molecules 2014, 19, 15213-15223. [CrossRef]

12. Bak, M.J.; Truong, V.L.; Kang, H.S.; Jun, M.; Jeong, W.S. Anti-inflammatory effect of procyanidins from wild grape (Vitis amurensis) seeds in LPS-induced RAW 264.7 cells. Oxidative Med. Cell. Longev. 2013, 2013, 409321. [CrossRef] [PubMed]

13. Pervaiz, S. Resveratrol: From grapevines to mammalian biology. FASEB J. 2003, 17, 1975-1985. [CrossRef] [PubMed]

14. Roemer, K.; Mahyar, R.M. The basis for the chemopreventive action of resveratrol. Drug Today 2002, 38, 571-580. [CrossRef]

15. Jeong, H.Y.; Kim, J.Y.; Lee, H.K.; Ha, D.T.; Song, K.S.; Bae, K.; Seong, Y.H. Leaf and stem of Vitis amurensis and its active components protect against amyloid beta protein (25-35)-induced neurotoxicity. Arch. Pharm. Res. 2010, 33, 1655-1664. [CrossRef]

16. Xuan, L.; Jiang, R.; Wu, Z.; Yi, H.; Yao, C.; Hou, Q.; Qu, C. Vam3, a compound derived from Vitis amurensis Rupr., attenuated colitis-related tumorigenesis by inhibiting NF-kappaB signaling pathway. Front. Pharmacol. 2016, 7, 311-315. [CrossRef] [PubMed]

17. Hien, T.T.; Oh, W.K.; Quyen, B.T.; Dao, T.T.; Yoon, J.H.; Yun, S.Y.; Kang, K.W. Potent vasodilation effect of amurens in G is mediated through the phosphorylation of endothelial nitric oxide synthase. Biochem. Pharmacol. 2012, 84, 1437-1450. [CrossRef] [PubMed]

18. Zhu, L.; Zhang, Y.; Lu, J. Phenolic contents and compositions in skins of red wine grape cultivars among various genetic backgrounds and originations. Int. J. Mol. Sci. 2012, 13, 3492-3510. [CrossRef] 
19. De la Cruz, A.A.; Ghislaine, H.; Céline, R.; Virginie, M.; Nathalie, O.; Louis, B.; Stéphane, D.; Delaunay, J.C.; Serge, D.; Jean, M.M.; et al. Anthocyanin identification and composition of wild Vitis spp. accessions by using LC-MS and LC-NMR. Anal. Chim. Acta 2012, 732, 145-152. [CrossRef] [PubMed]

20. Liang, Z.H.; Wu, B.H.; Fan, P.G.; Yang, C.X.; Duan, W.; Zheng, X.B.; Liu, C.Y.; Li, S.H. Anthocyanin composition and content in grape berry skin in Vitis germplasm. Food Chem. 2008, 111, 837-844. [CrossRef]

21. Zhao, Q.; Duan, C.Q.; Wang, J. Anthocyanins profile of grape berries of Vitis amurensis, its hybrids and their wines. Int. J. Mol. Sci. 2010, 11, 2212-2228. [CrossRef]

22. Margaret, A.C.; Marjoire, C.K.; Jimmy, S. Anthocyanin, phenolic composition, colour measurement and sensory analysis of BC commercial red wines. Food Res. Int. 2007, 40, 92-100.

23. Zhu, L.; Zhang, Y.L.; Zhang, W.; Lu, J. Effects of exogenous abscisic acid on phenolic characteristics of red Vitis vinifera grapes and wines. Food Sci. Biotechnol. 2016, 25, 361-370. [CrossRef]

24. Singleton, V.L.; Rossi, J.A. Colorimetry of total phenolics with phosphomolybdic-phosphotungstic acid reagents. Am. J. Enol. Viticult. 1965, 16, 144-158.

25. Dewanto, V.; Wu, X.; Adom, K.K.; Liu, R.H. Thermal processing enhances the nutritional values of tomatoes by increasing the total antioxidant activity. J. Agric. Food Chem. 2002, 50, 3010-3014. [CrossRef]

26. Sun, B.; Ricardo, J.M.; Spranger, I. Critical factors of vanillin assay for catechins and proanthocyanidins. J. Agric. Food Chem. 1998, 46, 4267-4274. [CrossRef]

27. Brand-Williams, W.; Cuvelier, M.E.; Berset, C. Use of a free-radical method to evaluate antioxidant activity. LWT Food Sci. Technol. 1995, 28, 25-30. [CrossRef]

28. Re, R.; Pellegrini, N.; Proteggente, A.; Pannala, A.; Yang, M.; Rice, E.C. Antioxidant activity applying an improved ABTS radical cation decolorization assay. Free Radic. Biol. Med. 1999, 26, 1231-1237. [CrossRef]

29. Benzie, I.F.F.; Strain, J.J. Ferric reducing ability of plasma (FRAP) as a measure of antioxidant power: The FRAP assay. Anal. Biochem. 1996, 239, 70-76. [CrossRef]

30. Xu, C.; Zhang, Y.; Cao, L. Phenolic compounds and antioxidant properties of different grape cultivars grown in China. Food Chem. 2010, 119, 1557-1565. [CrossRef]

31. Mazzuca, P.; Ferranti, P.; Picariello, G.; Chianese, L.; Addeo, F. Mass spectrometry in the study of anthocyanins and their derivatives: Differentiation of Vitis vinifera and hybrid grapes by liquid chromatography electrospray ionization mass spectrometry and tandem mass spectrometry. Mass Spectrom. Rev. 2005, 40, 83-90. [CrossRef]

32. Gianluca, P.; Pasquale, F.; Lina, C.; Francesco, A. Differentiation of Vitis vinifera L. and Hybrid Red Grapes by Matrix-Assisted Laser Desorption/Ionization Mass Spectrometry Analysis of Berry Skin Anthocyanins. J. Agric. Food Chem. 2012, 60, 4559-4566.

33. Nørbæk, K.; Brandt, J.K.; Nielsen, M.; Ørgaard, N.J. Flower pigment composition of crocus species and cultivars used for a chemotaxonomic investigation. Biochem. Syst. Ecol. 2002, 30, 763-791. [CrossRef]

34. Alcalde, E.; María, T.E.B.; Celestino, S.B.; Julián, C.R.G. Changes in the detailed pigment composition of red wine during maturity and ageing: A comprehensive study. Anal. Chim. Acta 2006, 563, 238-254. [CrossRef]

35. Liang, Z.C.; Owens, H.L.; Zhong, G.Y.; Cheng, L.L. Polyphenolic profiles detected in the ripe berries of Vitis vinifera germplasm. Food Chem. 2011, 129, 940-950. [CrossRef] [PubMed]

36. Castillo, M.N.; Fernandez, G.M.; Gomez, A.S.; Garcia, R.E.; Hermosin, G.I. Red-colour related phenolic composition of Garnacha Tintorera (Vitis vinifera L.) grapes and red wines. J. Agric. Food Chem. 2009, 57, 7883-7891. [CrossRef] [PubMed]

37. He, F.; He, J.J.; Pan, Q.H.; Duan, C.Q. Mass-spectrometry evidence confirming the presence of pelargonidin-3-O-glucoside in the berry skins of Cabernet Sauvignon and Pinot Noir (Vitis vinifera L.). Aust. J. Grape Wine Res. 2010, 16, 464-468. [CrossRef]

38. Sandhu, A.K.; Gu, L.W. Antioxidant capacity, phenolic content, and profiling of phenolic compounds in the seeds, skin, and pulp of Vitis rotundifolia (Muscadine Grapes) as determined by HPLC-DAD-ESI-MS. Agric. Food Chem. 2010, 58, 4681-4692. [CrossRef] [PubMed]

39. He, F.; Liang, N.N.; Mu, L.; Pan, Q.H.; Wang, J.; Reeves, M.J.; Duan, C.Q. Anthocyanins and their variation in red wines I. monomeric anthocyanins and their color expression. Molecules 2012, 17, 1571-1601. [CrossRef]

40. Tanaka, Y.; Brugliera, F. Flower colour and cytochromes P450. Phil. Trans. R. Soc. B 2013, 368, 20120432. [CrossRef]

41. Liang, Z.C.; Yang, Y.Z.; Cheng, L.L.; Zhong, G.Y. Polyphenolic composition and content in the ripe berries of wild Vitis species. Food Chem. 2012, 132, 730-738. [CrossRef]

42. Benbouguerra, N.; Richard, T.; Saucier, C.; Garcia, F. Voltammetric behavior, flavanol and anthocyanin contents, and antioxidant capacity of grape skins and seeds during ripening (Vitis vinifera var. Merlot, Tannat, and Syrah). Antioxidants 2020, 9, 800. [CrossRef]

43. Yang, Y.; Labate, J.A.; Liang, Z. Multiple loss-of-function 5-O-glucosyltransferase alleles revealed in Vitis vinifera, but not in other Vitis species. Theor. Appl. Genet. 2014, 127, 2433-2451. [CrossRef] [PubMed]

44. Song, R.G.; Lu, W.P.; Wang, J.; Shen, Y.J.; Lin, X.G.; Ge, Y.X.; Li, X.H.; Sun, K. Achievements and prospect on wine grape breeding in China. North. Hortic. 1996, 6, 3638.

45. Wang, J.; Zong, R.G.; Yin, L.R.; Sun, K.J.; Lin, X.G. ShuangFeng-A new hermaphrodite cultivar of Vitis amurensis Rupr. Acta Hortic. Sin. 1996, 23, 207.

46. Lin, X.G.; Zhang, H.; Xiu, J.C.; Feng, Y.C. Study on breeding of Shuangyou-A new hermaphrodite cultivar of Vitis amurensis Rupr. Sino-Overseas Grapevine Wine 1994, 4, 51-53. 
47. Song, R.G.; Lu, W.P.; Wang, J.; Shen, Y.J.; Shi, G.W. Studies on the breeding of shuang hong-A new cultivar of Vitis amurensis Rupr. Spec. Wild Econ. Anim. Plant Res. 1999, 1, 17-20.

48. Lu, W.P.; Wang, J.; Song, R.G.; Zang, Y.F.; Shen, Y.J.; Guo, Z.G.; Liu, Z.H.; Liu, J.K. Bereding research of cold-resistant grape variety of 'Zuohong No.1'. Sino-Overseas Grapevine Wine 2000, 1, 13-14.

49. Song, R.G.; Lu, W.P.; Guo, T.J.; Liu, J.K.; Shen, Y.J.; Lin, X.G.; Li, X.H.; Guo, Z.G. A new dry-red brewing grape variety 'Zuoyouhong'. Acta Hortic. Sin. 2005, 32, 757.

50. Song, R.G.; Lu, W.P.; Zhang, Q.T.; Li, X.H.; Yang, Y.M.; Fan, S.T.; Ai, J.; Shen, Y.J.; Lin, X.G. A new dry-red brewing grape variety 'Xuelanhong'. Spec. Wild Econ. Anim. Plant Res. 2012, 3, 14-19.

51. Li, S.C.; Wen, L.Z.; Zhang, F.Q.; Luo, F.M.; Yang, M.R.; Zhang, Y.Z.; Huang, D.F. A new cold-resistant and disease-resistant grape variety-Beichun. Chin. Bull. Bot. 1983, 2, 28-30.

52. Fan, P.G.; Li, S.C.; Wang, L.J.; Yang, M.R.; Wu, B.H.; Duan, W.; Li, L.S.; Zhong, J.Y.; Zhang, Y.Z.; Wen, L.Z.; et al. Breeding of new wine grapes of Beihong and Beimei. China Fruits 2010, 4, 5-8.

53. Fruit Research Institute of Jilin Academy of Agricultural Sciences. Introduction of new wine grape cultivars. Food Ferment. Ind. 1978, 6, 49-51.

54. Yang, J.H.; Wu, D.L. Grape cold resistance breeding. China Fruits 1959, 3, 22-24.

55. Song, R.G.; Lu, W.P.; Shen, Y.J.; Jin, R.H.; Jin, R.H.; Li, X.H.; Guo, Z.G.; Liu, J.K.; Lin, X.G. A new ice-red brewing grape cultivar ‘Beibinghong'. Acta Hortic. Sin. 2008, 35, 1085.

56. Zhuang, Y. Study on the Physiological Ecological Characteristics of Eight Vines. Master's Thesis, Anhui Agricultural University, Hefei, China, 2008.

57. Jie, M. The Study on Polyploid Breeding Technology of V. amurensis. Master's Thesis, Jilin Agricultural University, Changchun, China, 2008.

58. Pantelić, M.M.; Dabić Zagorac, D.Č.; Davidović, S.M.; Todić, S.R.; Bešlić, Z.S.; Gašić, U.M.; Tešić, Ž.L.; Natić, M.M. Identification and quantification of phenolic compounds in berry skin, pulp, and seeds in 13 grapevine varieties grown in Serbia. Food Chem. 2016, 211, 243-252.

59. Yue, Q.Y.; Xu, L.L.; Xiang, G.Q.; Yu, X.; Yao, Y.X. Characterization of gene expression profile, phenolic composition, and antioxidant capacity in red-fleshed grape berries and their wines. J. Agric. Food Chem. 2018, 66, 7190-7199. [CrossRef]

60. Robinson, W.B.; Weirs, L.D.; Bertino, J.J.; Mattick, L.R. The relation of anthocyanin composition to color stability of New York State wines. Am. J. Enol. Vitic. 1966, 17, 178-184.

61. Jánváry, L.; Hoffmann, T.; Pfeiffer, J.; Hausmann, L.; Töpfer, R.; Fischer, T.C.; Schwab, W.A. Double mutation in the anthocyanin 5-Oglucosyltransferase gene disrupts enzymatic activity in Vitis vinifera L. J. Agric. Food Chem. 2009, 57, 3512-3518. [CrossRef]

62. He, F.; Chen, W.K.; Yu, K.J.; Ji, X.N.; Duan, C.Q.; Reeves, M.J.; Wang, J. Molecular and biochemical characterization of the UDP-glucose:Anthocyanin 5-O-glucosyltransferase fromVitis amurensis. Phytochemistry 2015, 117, 363-372. [CrossRef]

63. Xing, R.R.; Li, S.Y.; He, F.; Yang, Z.; Duan, C.Q.; Li, Z.; Wang, J.; Pan, Q.H. Mass spectrometric and enzymatic evidence confirm the existence of anthocyanidin 3,5-O-diglucosides in cabernet sauvignon (Vitis vinifera L.) grape berries. Agric. Food Chem. 2015, 63, 3251-3260. [CrossRef] [PubMed] 